\title{
RESISTANCE TRAINING DOES NOT INDUCE UNIFORM ADAPTATIONS TO
}

\section{QUADRICEPS MUSCLES}

$4 \quad$ Wells $^{2}$, Adam R. Jajtner ${ }^{5}$, Kyle S. Beyer ${ }^{2}$, Carleigh H. Boone², Michael B. La Monica², Jeffrey

${ }^{1}$ Exercise Science and Sport Management, Kennesaw State University, Kennesaw, Georgia

$7 \quad{ }^{2}$ Institute of Exercise Physiology and Wellness, University of Central Florida, Orlando, Florida

$8 \quad{ }^{3}$ Department of Health Professions, Hofstra University, Hempstead, New York

$9 \quad{ }^{4}$ Exercise and Nutrition Science, Lipscomb University, Nashville, Tennessee

$10 \quad{ }^{5}$ Exercise Science/Physiology, Kent State University, Kent, Ohio

$11{ }^{6}$ Health \& Exercise Science, The College of New Jersey, Ewing, New Jersey

12 Running Title: Non-uniform adaptations to muscle following resistance training

13 Address for correspondence

14 Gerald T. Mangine, Ph.D.

15 Exercise Science and Sport Management

$16 \quad$ Kennesaw State University

17520 Parliament Garden Way NW

$18 \quad$ Kennesaw, GA 30144

19 Tel: $470-578-3425$

20 gmangine@kennesaw.edu 
Non-uniform adaptations to muscle following resistance training 2

23 Resistance training may differentially affect morphological adaptations along the length of uni-

24 articular and bi-articular muscles. The purpose of this study was to compare changes in muscle

25 morphology along the length of the rectus femoris (RF) and vastus lateralis (VL) in response to

26 resistance training. Following a 2 -wk preparatory phase, 15 resistance-trained men $(24.0 \pm 3.0$ y,

$2790.0 \pm 13.8 \mathrm{~kg}, 174.9 \pm 20.7 \mathrm{~cm})$ completed pre-training (PRE) assessments of muscle thickness

28 (MT), pennation angle (PA), cross-sectional area (CSA), and echo-intensity in the RF and VL at

2930,50 , and 70\% of each muscle's length; fascicle length (FL) was estimated from respective

30 measurements of MT and PA within each muscle and region. Participants then began a high

31 intensity, low volume ( 4 × $3-5$ repetitions, 3 min rest) lower-body resistance training program,

32 and repeated all PRE-assessments after 8 weeks $\left(2 \mathrm{~d} \cdot \mathrm{wk}^{-1}\right)$ of training (POST). Although three-

33 way (muscle $[\mathrm{RF}, \mathrm{VL}] \mathrm{x}$ region $[30,50,70 \%] \mathrm{x}$ time [PRE, POST]) repeated measures analysis

34 of variance did not reveal significant interactions for any assessment of morphology, significant

35 simple (muscle $\mathrm{x}$ time) effects were observed for CSA $(\mathrm{p}=0.002)$ and FL $(\mathrm{p}=0.016)$.

36 Specifically, average CSA changes favored the VL $\left(2.96 \pm 0.69 \mathrm{~cm}^{2}, \mathrm{p}<0.001\right)$ over the RF

$37\left(0.59 \pm 0.20 \mathrm{~cm}^{2}, \mathrm{p}=0.011\right)$, while significant decreases in average FL were noted for the RF (-

$381.03 \pm 0.30 \mathrm{~cm}, \mathrm{p}=0.004)$ but not the $\mathrm{VL}(-0.05 \pm 0.36 \mathrm{~cm}, \mathrm{p}=0.901)$. No other significant

39 differences were observed. The findings of this study demonstrate the occurrence of non-

40 homogenous adaptations in RF and VL muscle size and architecture following 8 weeks of high-

41 intensity resistance training in resistance-trained men. However, training does not appear to

42 influence region-specific adaptations in either muscle.

43 Keywords: regional hypertrophy; ultrasound; cross-sectional area; fascicle length; pennation

44 angle; resistance-trained adults 

aCC-BY 4.0 International license.

Non-uniform adaptations to muscle following resistance training 3

\section{Introduction}

Exercise selection and modality influence the degree to which specific muscles are

47 activated during training. Activation increases when exercises become more complex (1), while

48 the range of motion may alter the percent contribution of various muscle groups associated with

49 the exercise (2). These differences appear to be modulated by each muscle's specific role during

50 movement. For instance, the bi-articular $m$. rectus femoris (RF) and the mono-articular $m$. vastus

51 lateralis (VL) possess a similar function during knee extension, and thus, are similarly activated

52 during that exercise (3). However, their functional roles are different when an exercise requires

53 simultaneous motion at the hip and knee joints (e.g., back squat or deadlift) (4). During the

54 descent phase of the squat or deadlift, proximal RF fibers shorten to flex the hip, while VL and

55 distal RF fibers lengthen to flex the knee. This process is then reversed during the ascent. Since

56 the relative intensity (i.e., percent of maximal strength) will vary throughout dynamic motion,

57 due to changes in velocity and mechanical advantage $(4,5)$, it is possible that the degree of

58 stimulus exposure is also different between the RF and VL. Indeed, RF activation has been

59 observed to be $32 \%$ greater during the ascent phase of a squat compared to the descent, whereas

60 VL contribution remained consistent (6). Relative force production also appears to be different

61 between the RF and VL during the squat (7), though this has not been statistically assessed.

62 Consequently, these acute differences may affect training adaptations.

63 Adaptations to skeletal muscle are thought to be specific to the imposed demand of

64 exercise $(5,8)$ with changes in its metabolic and structural composition mirroring functional

65 requirements (9). Activated skeletal muscle fibers will hypertrophy in response to the mechanical

66 stress and fatigue induced by repeated training sessions $(10,11)$. However, uniform growth

67 throughout each muscle cannot be expected. Architectural changes have been observed to vary 
Non-uniform adaptations to muscle following resistance training 4

between muscles $(12,13)$, as well as across the width $(14,15)$ and length $(12,13)$ of specific muscles. These differences appear to be affected by training modality and potentially training experience. In untrained men, Narici and colleagues (1996) reported hypertrophy differences between each of the quadriceps muscles following 6-months of unilateral leg extensions

72 performed every other day, and that changes favored the most distal portions of the RF and VL.

73 Likewise, serial sarcomere additions (or losses) have been noted to occur across the width and

74 depth of the tibialis anterior following 6 weeks of eccentric training using various starting

75 positions (i.e., degree of plantar flexion) in rabbits (15). In contrast, greater VL hypertrophy

76 (middle to distal regions) compared to limited RF hypertrophy has been documented in

77 untrained, older women when training included both single- and a multi-joint exercise (i.e., leg

78 press) (13). Although these findings highlight the occurrence of non-homogenous adaptations

79 throughout skeletal muscle, uniform changes have also been documented following a similar

80 training protocol (i.e., 5 weeks of leg extensions) (16). These findings may be limited by

81 participant training experience and the simplicity of each study's respective programming.

82 Greater medial ( $5 \mathrm{~cm}$ from midline) adaptations in VL thickness (and possibly pennation angle)

83 compared to those found at the midline were observed following a 15-week, periodized, mixed-

84 method (i.e., resistance, Olympic and plyometric training) protocol (14). Still, programming was

85 meant to develop strength and power for sports performance in Division I soccer athletes; muscle

86 hypertrophy was a secondary training goal. Thus, it remains unclear whether changes in muscle

87 architecture would be homogenous following a protocol designed for muscle growth in trained

88 individuals. 
Non-uniform adaptations to muscle following resistance training 5

91 durable against future damage brought on by the same stimulus (18). This effect is more

92 pronounced in untrained lifters because most training designs are novel to this population and

93 their muscle fibers have yet to develop a "resistance" to various stimuli. Therefore, slight

94 differences in programming may not alter the training response. For instance, the addition of

95 single-joint exercises (i.e., triceps extension and elbow flexion) to a multi-joint resistance

96 training program did not result in greater hypertrophy for untrained men (19). Conversely,

97 greater precision in programming characteristics (i.e., intensity, volume, density) is needed for

98 initiating this process in trained adults $(5,8)$. For these individuals, set frequency $(20)$, training

99 intensity and volume $(21,22)$ and rest intervals $(23)$ have all been found to influence the

100 hypertrophy response. However, in several cases, the observed hypertrophy was not consistent

101 across each site (21-23), nor were these differences compared. Therefore, the purpose of this

102 study was to compare changes between RF and VL architecture along their longitudinal axis

103 following 8 weeks of resistance training in resistance-trained men. Based on previous reports

$104(12-14,24)$, we hypothesized that architectural changes would be different between the RF and

$105 \mathrm{VL}$, and that these changes would vary along each muscle's length.

\section{Materials and Methods}

\section{Study Design}

Reductions in skeletal muscle characteristics (i.e., size and architecture) may occur within

110 as little as 2 weeks of detraining (i.e., cessation of training) in resistance-trained populations

111 (25). Therefore, to assess the effect of training on muscular adaptations across RF and VL

112 regions, this study did not employ the use of a control group. Instead, a within-subjects design 
Non-uniform adaptations to muscle following resistance training 6

113 was used where pre-training (PRE) assessments of muscle morphology were compared to those

114 observed following 8 weeks of resistance training (POST). Initially, all participants reported to

115 the Human Performance Laboratory (HPL) to complete an obligatory 2-week preparatory

116 training program. Subsequently, PRE-assessments of muscle morphology were performed on all

117 participants. The participants then returned to the HPL on the following week (i.e., week 3) to

118 begin the 8-week training program. During the week following the 8 -week training intervention

119 (i.e., week 11), all PRE-assessments were repeated. Comparisons were made between muscles

120 and across regions over time.

\section{Participants}

122 Following an explanation of all procedures, risks, and benefits, 15 physically-active,

123 resistance-trained men $(24.0 \pm 3.0$ years; $90.0 \pm 13.8 \mathrm{~kg} ; 174.9 \pm 20.7 \mathrm{~cm})$ provided their

124 informed written consent to participate in this study. All participants were free of any physical

125 limitations (determined by medical history questionnaire and PAR-Q) and had been regularly

126 participating in resistance training for a minimum of 2 years $(5.7 \pm 2.2$ years $)$ at the time of

127 recruitment. Participants described their prior training habits to be different from the present

128 training regimen in terms of exercise order and groupings. Approximately $87 \%$ described their

129 normal repetition range to be higher (i.e., $6-12 \mathrm{RM}$ range) than the $3-5 \mathrm{RM}$ range used in this

130 study. Additionally, 47\% reported using shorter rest periods (i.e., $<3$ minutes), while $13.3 \%$ had

131 not tracked their rest times previously. The remaining participants employed a similar training

132 scheme to the program used in this study. This investigation was approved by the New England

133 Institutional Review Board. 
Non-uniform adaptations to muscle following resistance training 7

\section{Ultrasonography measurements}

Following 15 minutes of rest in the supine position, to allow for redistribution of body fluids (26), ultrasound images of the RF and VL were collected from the dominant thigh of each participant using a 12-MHz linear probe scanning head (General Electric LOGIQ P5, Wauwatosa, WI, USA). The same investigator identified all anatomical locations of interest using previously described landmark standards (26-28) to measure muscle thickness (MT; \pm 0.1 $\mathrm{cm}), \mathrm{CSA}\left( \pm 0.1 \mathrm{~cm}^{2}\right)$, echo intensity (EI; \pm 0.1 arbitrary units $\left.[\mathrm{au}]\right)$, and pennation angle (PA; $\pm 0.1^{\circ}$ ). For each muscle, images were collected from distal to proximal along the longitudinal distance of the midline at 30\% (i.e., Distal), 50\% (i.e., middle), and 70\% (i.e., proximal) of each muscle's length. For CSA and EI, the extended field of view mode $(\mathrm{Gain}=50 \mathrm{~dB}$; Depth $=5 \mathrm{~cm})$ was used to capture two consecutive panoramic images of the muscular regions of interest. For MT and PA, two images were collected from the same sites described for CSA and EI, but with the probe oriented longitudinal to the muscle tissue interface using Brightness Mode (B-mode) ultrasound. All collected images were transferred to a personal computer and analyzed by the same investigator using Image $\mathrm{J}$ (National Institutes of Health, Bethesda, MD, USA, version $1.45 \mathrm{~s})$.

The averaged values from both images for each measure within a specific region were used for statistical analysis. Fascicle length $(\mathrm{FL} ; \pm 0.1 \mathrm{~cm})$ for each muscle within each region was estimated using associated images for MT and PA. This methodology for determination of fascicle length has a reported estimated coefficient of variation of $4.7 \%$ (29) and can be found using the following equation (29-31): Fascicle $=\mathrm{MT} \cdot \operatorname{SIN}(\mathrm{PA})^{-1}$. The reliability of these procedures for assessing $\mathrm{MT}\left(\mathrm{ICC}_{3, \mathrm{~K}}=0.88-0.92, \mathrm{SEM}_{3, \mathrm{~K}}=0.15-0.39 \mathrm{~cm}\right), \mathrm{CSA}\left(\mathrm{ICC}_{3, \mathrm{~K}}=\right.$ $\left.0.88-0.99, \mathrm{SEM}_{3, \mathrm{~K}}=0.81-2.38 \mathrm{~cm}^{2}\right), \mathrm{EI}\left(\mathrm{ICC}_{3, \mathrm{~K}}=0.74-0.95, \mathrm{SEM}_{3, \mathrm{~K}}=2.59-6.44 \mathrm{au}\right), \mathrm{PA}$ 
Non-uniform adaptations to muscle following resistance training 8

158

159

160

161

162

163

164

165

166

167

168

169

170

171

172

173

174

175

$\left(\mathrm{ICC}_{3, \mathrm{~K}}=0.81-0.97, \mathrm{SEM}_{3, \mathrm{~K}}=0.27-1.44^{\circ}\right)$, and FL $\left(\mathrm{ICC}_{3, \mathrm{~K}}=0.81-0.96, \mathrm{SEM}_{3, \mathrm{~K}}=0.74-\right.$

$1.35 \mathrm{~cm}$ ) at $30 \%, 50 \%$, and $70 \%$ of the RF and VL length had been previously determined in 10 active, resistance-trained men $(25.3 \pm 2.0$ years; $90.8 \pm 6.8 \mathrm{~kg} ; 180.3 \pm 7.1 \mathrm{~cm})$.

\section{Resistance training intervention}

The details of training and strength testing have been described elsewhere (21). Briefly, all participants completed a 2 -week preparatory phase prior to the 8 -week intervention to familiarize them with the training exercises, protocol, and proper lifting technique. Performance during this phase, along with one-repetition maximum (1RM) strength assessed in the back squat, was used to calculate the intensity loads used during the intervention. The high-intensity, low-volume training program (4 sets of $3-5$ repetitions, 3-minute rest intervals) used in this study included four closed-chain, lower-body exercises (i.e., barbell back squat, deadlift, barbell lunge, and leg press) that were performed on two training sessions per week. The initial intensity load was set at $90 \%$ of each participant's tested (back squat) or estimated 1RM (all other exercises) (32). Training loads were progressively increased when all prescribed repetitions for an exercise were achieved on two consecutive workouts. All participants were required to complete at least 14 (of 16) training sessions ( 87.5\%). All sessions were completed under the direct supervision of certified strength and conditioning specialists (CSCS).

\section{Nutrient intake and dietary analysis}

During the training intervention, the participants were instructed to maintain their normal dietary intake habits. Following each training session, participants were provided $\sim 235 \mathrm{~mL}$ of chocolate milk (170 calories; 2.5 g fat; 29 g carbohydrate; 9 g protein) or Lactaid ${ }^{\circledR}$ (150 calories;

$2.5 \mathrm{~g}$ fat; $24 \mathrm{~g}$ carbohydrate; $8 \mathrm{~g}$ protein) for lactose-intolerant participants. Total kilocalorie and 
Non-uniform adaptations to muscle following resistance training 9

180

181

182

183

184

185

186

187

188

189

190

191

192

193

194

195

196 differences could be considered real, changes were compared to their calculated minimal

197 difference (MD) (34) by creating a 95\% confidence interval (C.I.) about the standard error of the

198 measurement (SEM). MD was then calculated using the following equation (MD = SEM x 1.96

$199 \times \sqrt{2}$ ). Any change occurring within this confidence interval was interpreted as being consistent

200 with the measurement error of the test, while changes occurring outside of the interval reflect

201 real changes in body composition. All data are reported as mean \pm standard error (SE) of the 
Non-uniform adaptations to muscle following resistance training 10 noted where average PA increased $\left(\mathrm{F}=4.1, \mathrm{p}=0.063, \eta_{\mathrm{P}}^{2}=0.23\right)$ by $0.69 \pm 0.34^{\circ}(95 \%$ C.I. $=-$ $0.04-1.43)$ and average FL decreased $\left(\mathrm{F}=3.7, \mathrm{p}=0.076, \eta_{\mathrm{P}}^{2}=0.21\right)$ by $-0.54 \pm 0.28(95 \%$ C.I.

$209=-1.14-0.07)$. Marginal estimates for measures of muscle morphology across 8 -weeks of 210 training are presented in Table 1.

$213 \operatorname{MT}\left(\mathrm{F}=0.3, \mathrm{p}=0.741, \eta_{\mathrm{P}}^{2}=0.02\right), \operatorname{CSA}\left(\mathrm{F}=1.9, \mathrm{p}=0.189, \eta_{\mathrm{P}}^{2}=0.12\right), \mathrm{PA}(\mathrm{F}=0.3, \mathrm{p}=0.757$

$\left.214 \eta_{\mathrm{P}}^{2}=0.02\right)$, or FL $\left(\mathrm{F}=0.6, \mathrm{p}=0.544, \eta^{2}{ }_{\mathrm{P}}=0.04\right)$, though a trend for $\mathrm{EI}\left(\mathrm{F}=3.2, \mathrm{p}=0.057, \eta^{2}{ }_{\mathrm{P}}=\right.$

215 0.19) was noted. Exploratory post-hoc analysis revealed a significant increase in RF EI at 70\%

$216(3.70 \pm 1.09 \mathrm{au}, \mathrm{p}=0.004)$ but not at any other location.

A significant simple (muscle $\mathrm{x}$ time) effect was observed for CSA $(\mathrm{F}=14.1, \mathrm{p}=0.002$,

$\left.218 \eta_{\mathrm{P}}^{2}=0.50\right)$ where changes favored the VL $\left(2.96 \pm 0.69 \mathrm{~cm}^{2}, 95 \%\right.$ C.I. $=1.48-4.44 \mathrm{~cm}^{2}, \mathrm{p}<$

$2190.001)$ over the RF $\left(0.59 \pm 0.20 \mathrm{~cm}^{2}, 95 \%\right.$ C.I. $\left.=0.15-1.03 \mathrm{~cm}^{2}, \mathrm{p}=0.011\right)$. A significant 220 simple (muscle $x$ time) effect was also observed for $F L\left(F=7.5, p=0.016, \eta^{2} \mathrm{p}=0.35\right)$ where $R F$ 221 decreased $(-1.03 \pm 0.30 \mathrm{~cm}, 95 \%$ C.I. $=-1.68--0.38 \mathrm{~cm}, \mathrm{p}=0.004)$ and VL did not change $(-$ $2220.05 \pm 0.36 \mathrm{~cm}, 95 \%$ C.I. $=-0.82-0.73 \mathrm{~cm}, \mathrm{p}=0.901)$. Additionally, trends were noted for MT $223\left(\mathrm{~F}=4.3, \mathrm{p}=0.058, \mathrm{\eta}_{\mathrm{P}}{ }_{\mathrm{P}}=0.23\right)$ and PA $\left(\mathrm{F}=3.3, \mathrm{p}=0.091, \mathrm{\eta}_{\mathrm{P}}^{2}=0.19\right)$. Differences between 
Non-uniform adaptations to muscle following resistance training 11

224 muscles and regions at PRE and POST for each assessment of muscle morphology are illustrated

225 in Figure 1.

Although, no other statistical differences were observed, a larger percentage of

232 larger percentage of participants experienced changes that exceeded the MD at 30\% (CSA, PA,

233 and FL) compared to other regions. In contrast, changes exceeding the MD for each VL region

234 varied by morphological assessment. Changes in MT (RF and VL) did not exceed the MD for

235 any region. Regional changes in VL and RF morphology and the percentage of participants exceeding each measure's respective MD are presented in Table 2.

[INSERT TABLE 2 HERE]

No differences in relative kilocalorie or protein intake across 8-weeks of resistance training were observed and have been previously reported elsewhere(21).

\section{Discussion}

243 across the RF and VL were uniform following 8 weeks of resistance training in resistance-trained

244 men. We hypothesized that changes would differ between these muscles, as well as along their

245 longitudinal axes, due to differences in their functional role during multi-joint, lower-body 
Non-uniform adaptations to muscle following resistance training 12

246

247

248

249

250

251

252

253

254

255

256

257

258

259

260

261

262

263

264

265

266

267

268

exercises (e.g., squat and deadlift) (4). Overall, we observed greater hypertrophy in the VL compared to the RF, which was consistent with what was observed by Häkkinen and colleagues (2001) but not others $(12,16,24)$. Following training that solely featured open-chain exercises (i.e., leg extensions), similar $(16)$ or greater $(12,24)$ RF hypertrophy, compared to the VL, had been noted. Additionally, we observed decreased FL and a trend for increased PA in the RF with no changes occurring in the VL. These findings differed from those of Seynnes and colleagues (2007) who reported uniform increases in FL and PA. Further, aside from a trend towards decreased proximal RF muscle quality (via EI), we found no region-specific differences in morphological adaptations. Previously, differences had been reported along the longitudinal axis $(12,13,24)$ following resistance training protocols that have either solely featured unilateral leg extensions $(12,24)$ or included only a single multi-joint exercise (13) in adults with limited training experience. However, our study appears to be the first to examine regional differences in muscular changes following a training program that solely used multiple-joint exercises in a group of resistance-trained men.

The quadriceps muscle group is comprised of four muscles that insert into the patella tendon but originate from various structures of the hip and femur. Due to differences in origination, their individual functions are affected by movement. During the concentric phase of a leg extension, the quadriceps muscles are activated together to equally contribute to force production $(35,36)$; though RF activation may increase during the eccentric phase (12). In contrast, RF activation is less than the VL's during a closed-chain exercise (e.g., leg press, squat) (35-37) and may also be less during the eccentric phase compared to the concentric phase (6). Previously, greater RF (than VL) hypertrophy has been found when training only included leg extensions $(12,24)$. However, our training protocol only included closed-chain exercises and 
Non-uniform adaptations to muscle following resistance training 13

269

270

271 potentially reduced RF quality, may have been related to our programming design being more

272 specific to VL activation. While this cannot be confirmed from our previous report of similar

273

274

275

276

277

278

279

280

281

282

283

284

285

286

287

288

289

290

291

resulted in greater VL hypertrophy. When training has previously included a closed-chain exercise, adaptations favored the VL (13). Thus, it is possible that greater VL adaptations, and changes in VL $\left(-1.62 \pm 1.80 \mathrm{~V} \cdot \sec \cdot \% 1 \mathrm{RM}^{-1}\right)$ and $\mathrm{RF}\left(-1.72 \pm 1.47 \mathrm{~V} \cdot \mathrm{sec} \cdot \% 1 \mathrm{RM}^{-1}\right)$

activation across maximal and submaximal strength testing (21), activation was only assessed at $50 \%$ of muscle length. It remains unclear whether adaptational differences in activation exist along the length of each muscle following this type of programming in trained individuals.

In addition to hypertrophy differences, differences in architectural changes were also seen between muscles. Increased PA and decreased FL occurred in the RF while no changes were seen in the VL. Changes in muscle size are thought to affect muscle architecture $(14,24,38-40)$. Specifically, increased MT has been associated with increased PA $(24,38,39)$ but not FL $(14$, 24, 39), though individuals who possess greater CSA have been found to have greater PA and FL (40). Following resistance exercise, damaged areas of muscle are inhabited by satellite cells, which fuse to the existing muscle tissue (41) and add new contractile proteins that increase the diameter of existing sarcomeres and length of fibers (17). Although changes in muscle thickness and fiber diameter should affect fiber orientation and insertion angle, changes in FL may be dependent upon exercise modality. When training is predominantly comprised of musclelengthening actions (i.e., eccentric training), a greater number of sarcomeres are added in serial fashion compared to concentric-only or mixed contractions $(42,43)$. Here, the training protocol included exercises that incorporated both eccentric and concentric contractions. However, the degree and duration of eccentric tension may have varied based on individual technique (e.g., speed of lowering the bar during the deadlift, degree of hip extensor involvement, range of 
Non-uniform adaptations to muscle following resistance training 14

292

293

294

295

296

297

298

299

300

301

302

303

304

305

306

307

308

309

310

311

312

313

314

motion). This variability was reflected in the standard errors for FL changes being larger than their respective means, as well as in the percentage of participants exceeding the MD needed to observe "real" changes at each measurement site (see Table 2). It is also possible that FL adaptations were missed because measurements used for FL estimation were collected along the midline of each muscle. Previously, Wells and colleagues (2014) reported differences between FL changes observed along the VL midline (at 50\% muscle length) and a site located $5 \mathrm{~cm}$ medially. It is possible that our training protocol, how participants performed the exercises, and the specific sites used for FL estimation, limited our potential for observing improvements in FL. Aside from a trend towards increased EI (at proximal RF), our data did not support our hypothesis that adaptations would differ between muscle regions. Previously, morphological changes along the longitudinal axis of the RF and VL have been reported to be equivocal $(12,13$, $16,24)$ when multi-joint exercises are used sparingly or are non-existent in adults with limited training experience. When programming only included leg extensions, hypertrophy has been found to be greater in the distal quadriceps regions after 3-6 months of training $(12,24)$. Conversely, Häkkinen and colleagues (2001) noted similar hypertrophy along the length of the quadriceps, but not when individual muscles were considered. Interestingly, Seynnes and colleagues (2007) reported no differences between the distal region and muscle belly. However, those findings may have been limited by a much shorter training period (i.e., 5 weeks) and an inappropriate statistical analysis (i.e., separate paired t-Tests). As we have previously discussed, the lack of regional differences may be related to quadriceps recruitment during various exercise modalities. Quadriceps activation favors the distal regions during an open-chain, leg extension, whereas greater proximal activation occurs in tasks that require active hip flexion (44). During complex motions (e.g., walking) the contribution of proximal and distal RF regions have been 
Non-uniform adaptations to muscle following resistance training 15

315 shown to vary throughout the motion and in relation to velocity (45). Beyond these studies,

316 however, little is known about regional differences in quadriceps activation during multi-joint,

317 closed-chain exercises. It is possible that the trend observed in reduced proximal RF quality (i.e.,

318 increased EI) may be indicative of a detraining effect brought on by a reduced contribution from

319 this region throughout training.

320 The findings of this study demonstrate the occurrence of non-homogenous adaptations in

321 RF and VL morphology following 8 weeks of resistance training in resistance-trained men. The

322 training program resulted in greater VL hypertrophy, which may have been the consequence of

323 reduced RF contribution during closed-chain, multi-joint exercises. Further, the high degree of

324 variability in which these exercises can be performed (e.g., speed of lowering the bar during the

325 deadlift, degree of hip extensor involvement, range of motion) may have been responsible for the

326 observed increase in PA and decrease in FL of the RF. Contrary to our hypothesis, however, we

327 did not observe differences between regions (i.e., proximal, middle, and distal) of either muscle,

328 save for a trend in reduced proximal RF quality. Since little is known regarding region-specific

329 quadriceps activation during closed-chain, multi-joint exercises, it remains unclear why regional

330 adaptations were uniform. Nevertheless, it may be advisable for strength coaches and athletes to

331 incorporate hip flexion exercises within lower-body resistance training programs to avoid

332 potential reductions in proximal RF quality. 
Non-uniform adaptations to muscle following resistance training 16

\section{References}

335 1. Boudreau SN, Dwyer MK, Mattacola CG, Lattermann C, Uhl TL, McKeon JM. Hip-

336 muscle activation during the lunge, single-leg squat, and step-up-and-over exercises. Journal of

337 Sport Rehabilitation. 2009;18(1):91-103.

338 2. Caterisano A, Moss RE, Pellinger TK, Woodruff K, Lewis VC, Booth W, et al. The

339 effect of back squat depth on the EMG activity of 4 superficial hip and thigh muscles. J Strength

340 Cond Res. 2002;16(3):428-32.

341 3. Purkayastha S, Cramer JT, Trowbridge CA, Fincher AL, Marek SM. Surface

342 electromyographic amplitude-to-work ratios during isokinetic and isotonic muscle actions.

343 Journal of athletic training. 2006;41(3):314.

344 4. Floyd RT. Manual of Structural Kinesiology2014.

345 5. Haff GG, Triplett NT. Essentials of Strength Training and Conditioning 4th Edition:

346 Human kinetics; 2015.

347 6. McCaw ST, Melrose DR. Stance width and bar load effects on leg muscle activity during

348 the parallel squat. Med Sci Sports Exerc. 1999;31:428-36.

349 7. Escamilla RF, Fleisig GS, Zheng N, Lander JE, Barrentine SW, Andrews JR, et al.

350 Effects of technique variations on knee biomechanics during the squat and leg press. Med Sci

351 Sports Exerc. 2001;33(9):1552-66.

352 8. Ratamess NA, Alvar BA, Evetoch TK, Housh TJ, Kibler WB, J KW, et al. Progression

353 models in resistance training for healthy adults. Position Stand: Medicine and science in sports

354 and exercise. 2009;41(3):687-708.

355 9. Flück M. Functional, structural and molecular plasticity of mammalian skeletal muscle in 356 response to exercise stimuli. J Exp Biol. 2006;209(12):2239-48. 
Non-uniform adaptations to muscle following resistance training 17

357 10. Moritani T. Neuromuscular adaptations during the acquisition of muscle strength, power

358 and motor tasks. J Biomech. 1993;26:95-107.

359 11. Evans WJ. Effects of exercise on senescent muscle. Clin Orthop Relat Res. $360 \quad$ 2002;403:S211-S20.

361 12. Narici M, Hoppeler H, Kayser B, Landoni L, Claassen H, Gavardi C, et al. Human 362 quadriceps cross-sectional area, torque and neural activation during 6 months strength training.

363 Acta Physiol Scand. 1996;157(2):175-86.

364 13. Häkkinen K, Pakarinen A, Kraemer WJ, Häkkinen A, Valkeinen H, Alen M. Selective 365 muscle hypertrophy, changes in EMG and force, and serum hormones during strength training in 366 older women. J Appl Physiol. 2001;91(2):569-80.

367 14. Wells AJ, Fukuda DH, Hoffman JR, Gonzalez AM, Jajtner AR, Townsend JR, et al. 368 Vastus Lateralis Exhibits Non-Homogenous Adaptation to Resistance Training. Muscle Nerve. 3692014.

370 15. Butterfield TA, Herzog W. The magnitude of muscle strain does not influence serial 371 sarcomere number adaptations following eccentric exercise. Pflügers Archiv. 2006;451(5):688372700.

373 16. Seynnes OR, de Boer M, Narici MV. Early skeletal muscle hypertrophy and architectural 374 changes in response to high-intensity resistance training. J Appl Physiol. 2007;102(1):368-73.

375 17. Toigo M, Boutellier U. New fundamental resistance exercise determinants of molecular 376 and cellular muscle adaptations. Eur J Appl Physiol. 2006;97(6):643-63.

377 18. Anderson T, Kearney JT. Effects of three resistance training programs on muscular 378 strength and absolute and relative endurance. Res Q Exerc Sport. 1982;53(1):1-7. 
Non-uniform adaptations to muscle following resistance training 18

379 19. Gentil P, Soares SRS, Pereira MC, Cunha RRd, Martorelli SS, Martorelli AS, et al. Effect

380 of adding single-joint exercises to a multi-joint exercise resistance-training program on strength

381 and hypertrophy in untrained subjects. Applied Physiology, Nutrition, and Metabolism.

$382 \quad 2013 ; 38(3): 341-4$

383 20. Goto K, Nagasawa M, Yanagisawa O, Kizuka T, Ishii N, Takamatsu K. Muscular

384 adaptations to combinations of high-and low-intensity resistance exercises. J Strength Cond Res.

$385 \quad 2004 ; 18(4): 730-7$.

386 21. Mangine GT, Hoffman JR, Gonzalez AM, Townsend JR, Wells AJ, Jajtner AR, et al. The

387 effect of training volume and intensity on improvements in muscular strength and size in

388 resistance-trained men. Physiological Reports. 2015;3(8):e12472.

389 22. Schoenfeld BJ, Ratamess NA, Peterson MD, Contreras B, Sonmez G, Alvar BA. Effects

390 of different volume-equated resistance training loading strategies on muscular adaptations in

391 well-trained men. J Strength Cond Res. 2014;28(10):2909-18.

392 23. Schoenfeld BJ, Pope ZK, Benik FM, Hester GM, Sellers J, Nooner JL, et al. Longer

393 Interset Rest Periods Enhance Muscle Strength and Hypertrophy in Resistance-Trained Men. J

394 Strength Cond Res. 2016;30(7):1805-12.

395 24. Ema R, Wakahara T, Miyamoto N, Kanehisa H, Kawakami Y. Inhomogeneous 396 architectural changes of the quadriceps femoris induced by resistance training. Eur J Appl 397 Physiol. 2013;113(11):2691-703.

398 25. French D. Adaptations to anaerobic training programs. In: Haff GG, T T-M, editors.

399 Essentials of Strength Training and Conditioning. 4th ed. Champaign, IL: Human kinetics; 2015. 
Non-uniform adaptations to muscle following resistance training 19

400 26. Berg H, Tedner B, Tesch P. Changes in lower limb muscle cross-sectional area and tissue

401 fluid volume after transition from standing to supine. Acta Physiologica Scandinavica.

$402 \quad 1993 ; 148(4): 379-85$.

403 27. Bemben M. Use of diagnostic ultrasound for assessing muscle size. Journal of Strength \&

404 Conditioning Research. 2002;16(1):103-8.

405 28. Cadore EL, Izquierdo M, Conceição M, Radaelli R, Pinto RS, Baroni BM, et al. Echo 406 intensity is associated with skeletal muscle power and cardiovascular performance in elderly 407 men. Experimental Gerontology. 2012;47(6):473-8.

408 29. Kumagai K, Abe T, Brechue WF, Ryushi T, Takano S, Mizuno M. Sprint performance is 409 related to muscle fascicle length in male 100-m sprinters. J Appl Physiol. 2000;88(3):811-6.

410 30. Abe T, Brechue WF, Fujita S, Brown JB. Gender differences in FFM accumulation and 411 architectural characteristics of muscle. Med Sci Sports Exerc. 1998;30(7):1066-70.

412 31. Alegre LM, Jiménez F, Gonzalo-Orden JM, Martín-Acero R, Aguado X. Effects of 413 dynamic resistance training on fascicle lengthand isometric strength. J Sports Sci. $414 \quad 2006 ; 24(05): 501-8$.

415 32. Brzycki M. Strength testing-predicting a one-rep max from reps-to-fatigue. Journal of 416 Physical Education, Recreation \& Dance. 1993;64(1):88-90.

417 33. Cohen J. Statistical Power Analysis for the Behavioral Sciences1988. 284-8 p.

418 34. Weir JP. Quantifying test-retest reliability using the intraclass correlation coefficient and 419 the SEM. J Strength Cond Res. 2005;19(1):231-40.

420 35. Stensdotter A-K, Hodges P, Mellor R, Sundelin G, Häger-Ross C. Quadriceps activation 421 in closed and in open kinetic chain exercise. Med Sci Sports Exerc. 2003;35(12):2043-7. 
Non-uniform adaptations to muscle following resistance training 20

422 36. Ema R, Sakaguchi M, Akagi R, Kawakami Y. Unique activation of the quadriceps

423 femoris during single-and multi-joint exercises. Eur J Appl Physiol. 2016;116(5):1031-41.

424 37. Luera MJ, Stock MS, Chappell AD. Electromyographic amplitude vs. concentric and

425 eccentric squat force relationships for monoarticular and biarticular thigh muscles. J Strength

426 Cond Res. 2014;28(2):328-38.

427 38. Kawakami Y, Abe T, Fukunaga T. Muscle-fiber pennation angles are greater in 428 hypertrophied than in normal muscles. J Appl Physiol. 1993;74(6):2740-4.

429 39. Kawakami Y, Abe T, Kuno S-Y, Fukunaga T. Training-induced changes in muscle 430 architecture and specific tension. Eur J Appl Physiol Occup Physiol. 1995;72(1-2):37-43.

431 40. Ikegawa S, Funato K, Tsunoda N, Kanehisa H, Fukunaga T, Kawakami Y. Muscle force 432 per cross-sectional area is inversely related with pennation angle in strength trained athletes. J 433 Strength Cond Res. 2008;22(1):128-31.

434 41. Carosio S, Berardinelli MG, Aucello M, Musarò A. Impact of ageing on muscle cell 435 regeneration. Ageing research reviews. 2011;10(1):35-42.

436 42. Lynn R, Morgan D. Decline running produces more sarcomeres in rat vastus intermedius 437 muscle fibers than does incline running. J Appl Physiol. 1994;77(3):1439-44.

438 43. Lynn R, Talbot J, Morgan D. Differences in rat skeletal muscles after incline and decline 439 running. J Appl Physiol. 1998;85(1):98-104.

440 44. Watanabe K, Kouzaki M, Moritani T. Task-dependent spatial distribution of neural 441 activation pattern in human rectus femoris muscle. J Electromyogr Kinesiol. 2012;22(2):251-8.

442 45. Watanabe K, Kouzaki M, Moritani T. Regional neuromuscular regulation within human 443 rectus femoris muscle during gait. J Biomech. 2014;47(14):3502-8. 
Non-uniform adaptations to muscle following resistance training 21

\section{Figure Legends}

446 Figure 1. Regional and muscular differences in muscle morphology across 8-weeks of resistance 447 training. (A. Muscle thickness; B. Cross-sectional area; C. Echo-intensity; D. Pennation angle; 448 and E. Fascicle length)

449 Note: $*=$ Significant $(\mathrm{p}<0.05)$ difference between PRE and POST; $\uparrow=$ Significant $(\mathrm{p}<0.05)$ 450 differences between RF and VL; $\#=$ Difference $(\mathrm{p}<0.10)$ between PRE and POST; $\$=$ 451 Difference $(\mathrm{p}<0.10)$ between RF and VL; N.S. $=$ Not significant. 
Non-uniform adaptations to muscle following resistance training 22

Table 1. Changes in marginal estimates of combined RF and VL morphology following 8-weeks of resistance training (mean \pm SE).

\begin{tabular}{lcccccc}
\hline & PRE & $95 \%$ C.I. & POST & $95 \%$ C.I. & $\Delta$ & 95\% C.I. \\
\hline Muscle thickness $(\mathrm{cm})$ & $2.13 \pm 0.07$ & $(1.98-2.28)$ & $2.13 \pm 0.07$ & $(1.99-2.27)$ & $0.00 \pm 0.01$ & $(-0.02-0.02)$ \\
Cross-sectional area $\left(\mathrm{cm}^{2}\right)$ & $23.05 \pm 1.23$ & $(20.42-25.68)$ & $24.82 \pm 1.26^{*}$ & $(22.11-27.53)$ & $1.78 \pm 0.40$ & $(-2.63--0.92)$ \\
Echo intensity $(\mathrm{au})$ & $60.03 \pm 1.57$ & $(56.66-63.40)$ & $60.67 \pm 1.20$ & $(58.11-63.23)$ & $0.65 \pm 0.91$ & $(-2.59-1.30)$ \\
Pennation angle $\left({ }^{\circ}\right)$ & $13.05 \pm 0.37$ & $(12.27-13.84)$ & $13.74 \pm 0.34 \dagger$ & $(13.01-14.48)$ & $0.69 \pm 0.34$ & $(-1.43-0.04)$ \\
Fascicle length $(\mathrm{cm})$ & $9.84 \pm 0.36$ & $(9.07-10.62)$ & $9.31 \pm 0.31 \dagger$ & $(8.64-9.97)$ & $-0.54 \pm 0.28$ & $(-0.07-1.14)$ \\
\hline
\end{tabular}

Note: *Significantly $(\mathrm{p}<0.05)$ different from PRE; $\dagger$ Different $(\mathrm{p}<0.10)$ from PRE. 
Non-uniform adaptations to muscle following resistance training 23

Table 2. Percentage of participants exceeding the minimal difference for regional changes in muscle morphology following 8-weeks of resistance training (mean $\pm \mathrm{SE}$ ).

\begin{tabular}{|c|c|c|c|c|c|c|c|c|c|}
\hline & \multicolumn{3}{|c|}{ Distal } & \multicolumn{3}{|c|}{ Middle } & \multicolumn{3}{|c|}{ Proximal } \\
\hline & $\Delta$ & MD & $\begin{array}{c}\text { \%Exceeding } \\
\text { MD }\end{array}$ & $\Delta$ & $\mathrm{MD}$ & $\begin{array}{c}\text { \%Exceeding } \\
\text { MD }\end{array}$ & $\Delta$ & MD & $\begin{array}{c}\text { \%Exceeding } \\
\text { MD }\end{array}$ \\
\hline \multicolumn{10}{|l|}{ Muscle Thickness (cm) } \\
\hline Rectus Femoris & $-0.002 \pm 0.018$ & 0.62 & 0.0 & $-0.047 \pm 0.019$ & 0.45 & 0.0 & $-0.030 \pm 0.036$ & 1.03 & 0.0 \\
\hline Vastus Lateralis & $0.036 \pm 0.022$ & 0.48 & 0.0 & $0.031 \pm 0.020$ & 0.42 & 0.0 & $0.011 \pm 0.033$ & 0.38 & 0.0 \\
\hline \multicolumn{10}{|l|}{ Cross-sectional Area $\left(\mathrm{cm}^{2}\right)$} \\
\hline Rectus Femoris & $0.675 \pm 0.289$ & 2.20 & 13.3 & $0.446 \pm 0.340$ & 4.6 & 6.7 & $0.657 \pm 0.391$ & 3.69 & 0.0 \\
\hline Vastus Lateralis & $2.545 \pm 0.854$ & 3.99 & 33.3 & $4.283 \pm 1.345$ & 3.05 & 53.3 & $2.051 \pm 0.862$ & 6.44 & 6.7 \\
\hline \multicolumn{10}{|l|}{ Echo intensity (au) } \\
\hline Rectus Femoris & $-1.580 \pm 1.563$ & 14.27 & 0.0 & $2.075 \pm 1.223$ & 6.97 & 13.3 & $3.703 \pm 1.093$ & 16.38 & 0.0 \\
\hline Vastus Lateralis & $0.120 \pm 1.867$ & 7.55 & 20.0 & $-0.412 \pm 1.401$ & 6.47 & 20.0 & $-0.054 \pm 1.530$ & 9.26 & 20.0 \\
\hline \multicolumn{10}{|l|}{ Pennation angle $\left({ }^{\circ}\right)$} \\
\hline Rectus Femoris & $0.420 \pm 0.937$ & 3.16 & 33.3 & $2.020 \pm 0.577$ & 3.67 & 20.0 & $1.090 \pm 0.730$ & 3.46 & 13.3 \\
\hline Vastus Lateralis & $-0.027 \pm 0.609$ & 1.68 & 53.3 & $0.430 \pm 0.568$ & 1.91 & 20.0 & $0.223 \pm 1.099$ & 0.75 & 80.0 \\
\hline \multicolumn{10}{|l|}{ Fascicle length $(\mathrm{cm})$} \\
\hline Rectus Femoris & $-0.331 \pm 0.800$ & 2.94 & 33.3 & $-1.869 \pm 0.512$ & 3.21 & 13.3 & $-0.887 \pm 0.547$ & 3.62 & 13.3 \\
\hline Vastus Lateralis & $0.270 \pm 0.298$ & 1.90 & 6.7 & $-0.058 \pm 0.439$ & 2.73 & 20.0 & $-0.349 \pm 0.946$ & 3.16 & 40.0 \\
\hline
\end{tabular}

Note: $\Delta=$ PRE-POST changes; $\mathrm{MD}=$ Minimal Difference 
Figure 1. Regional and muscular differences in muscle morphology across 8-weeks of resistance training. (A. Muscle thickness; B. Cross-sectional area; C. Echo-intensity; D. Pennation angle; and E. Fascicle length)

A.

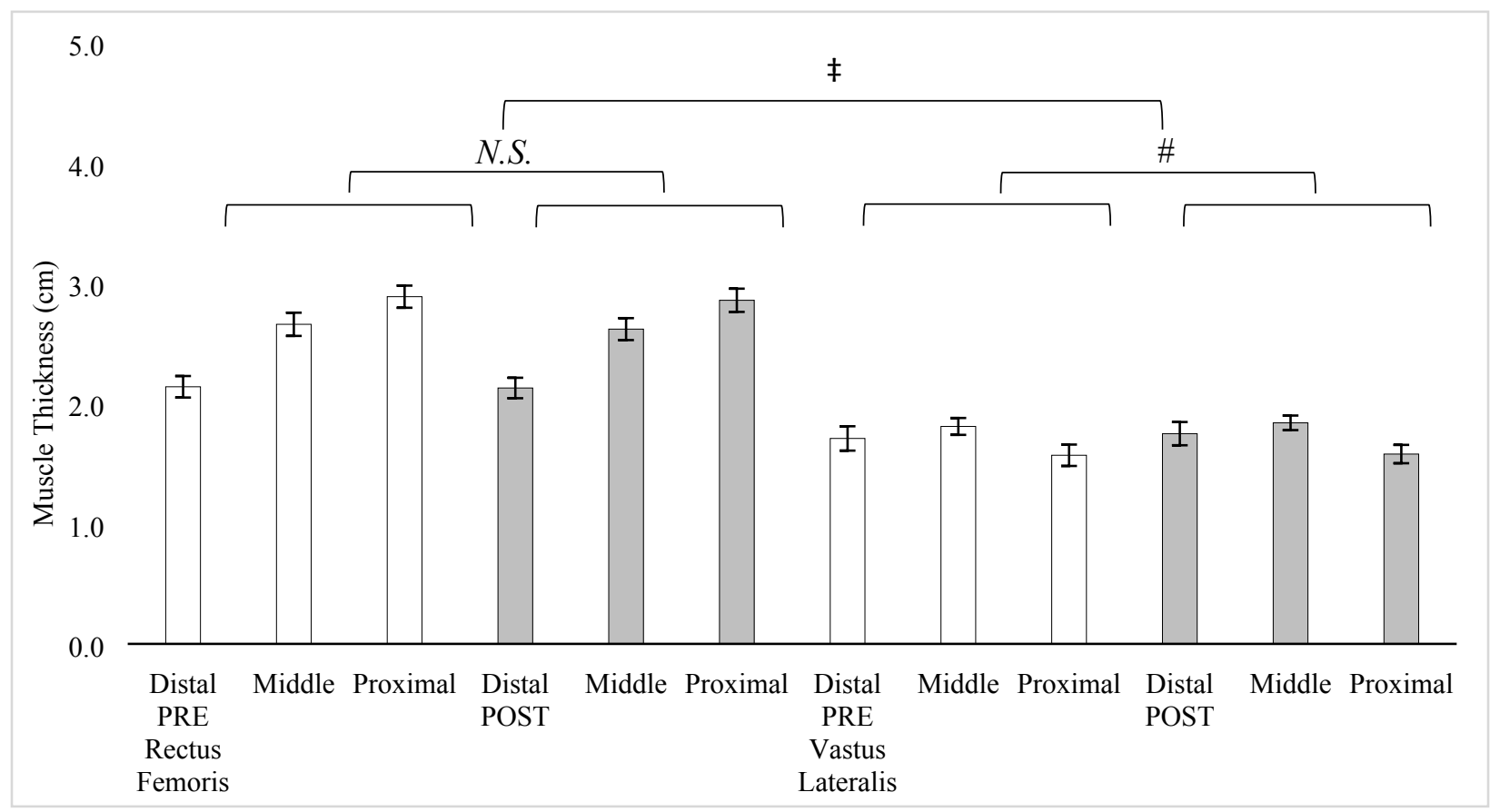


B.

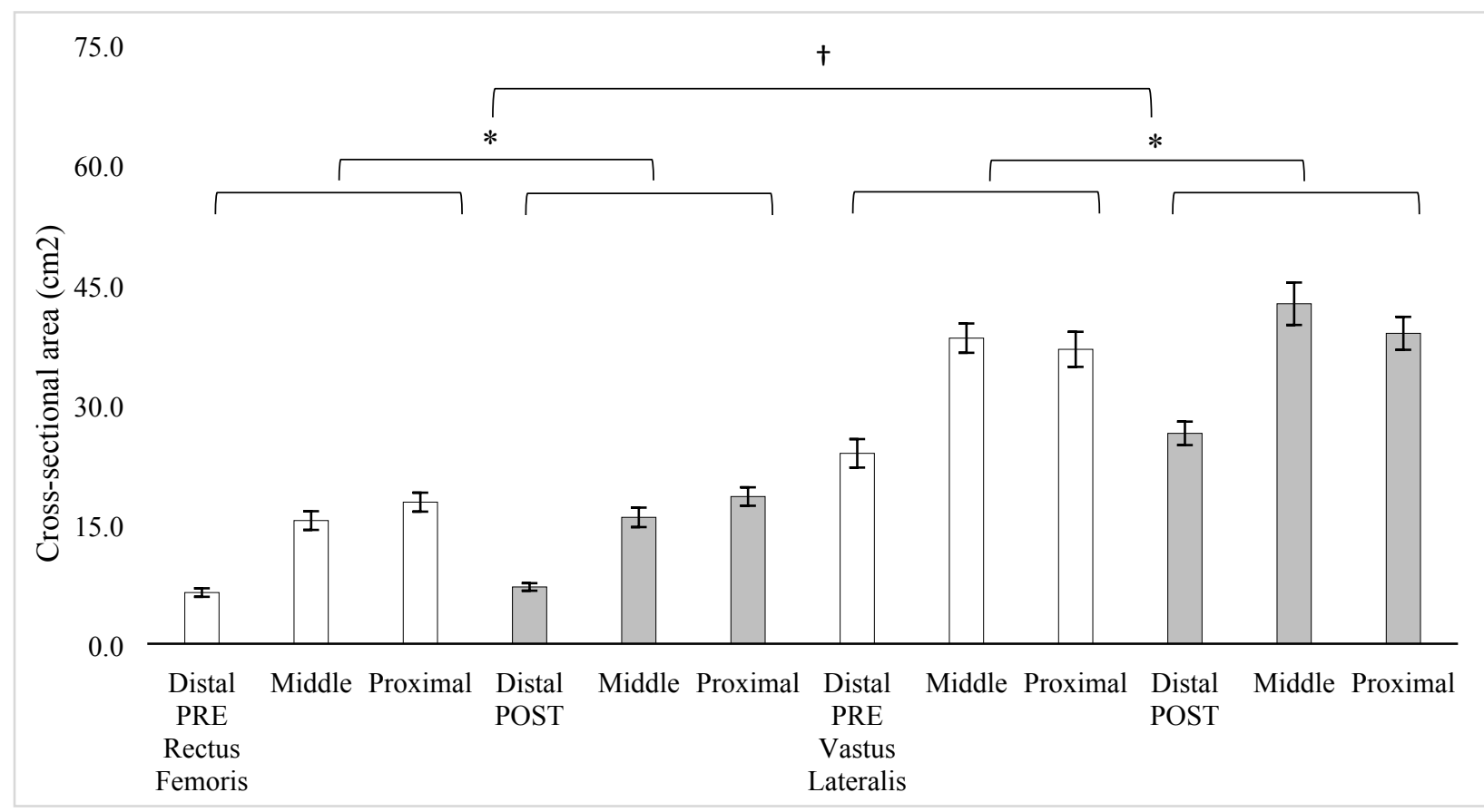

C.

100

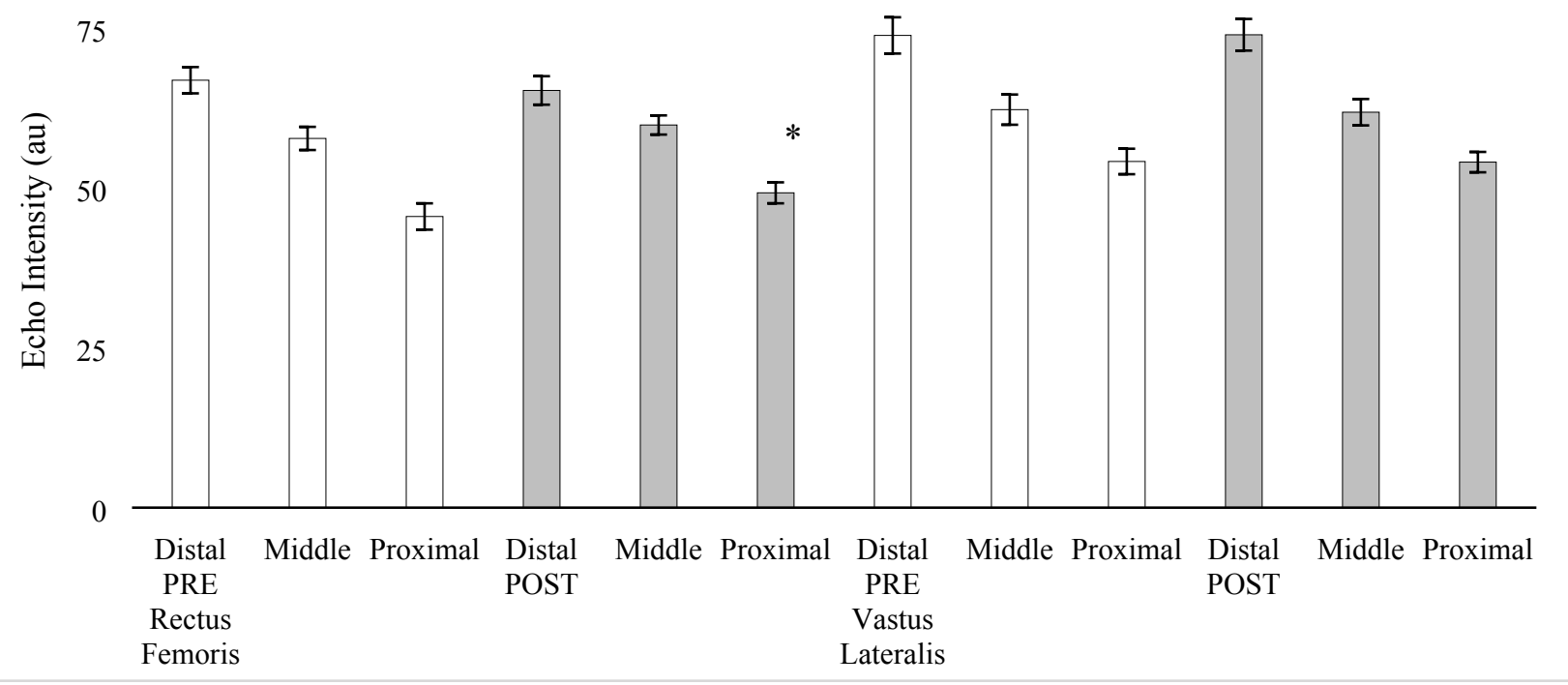


D.

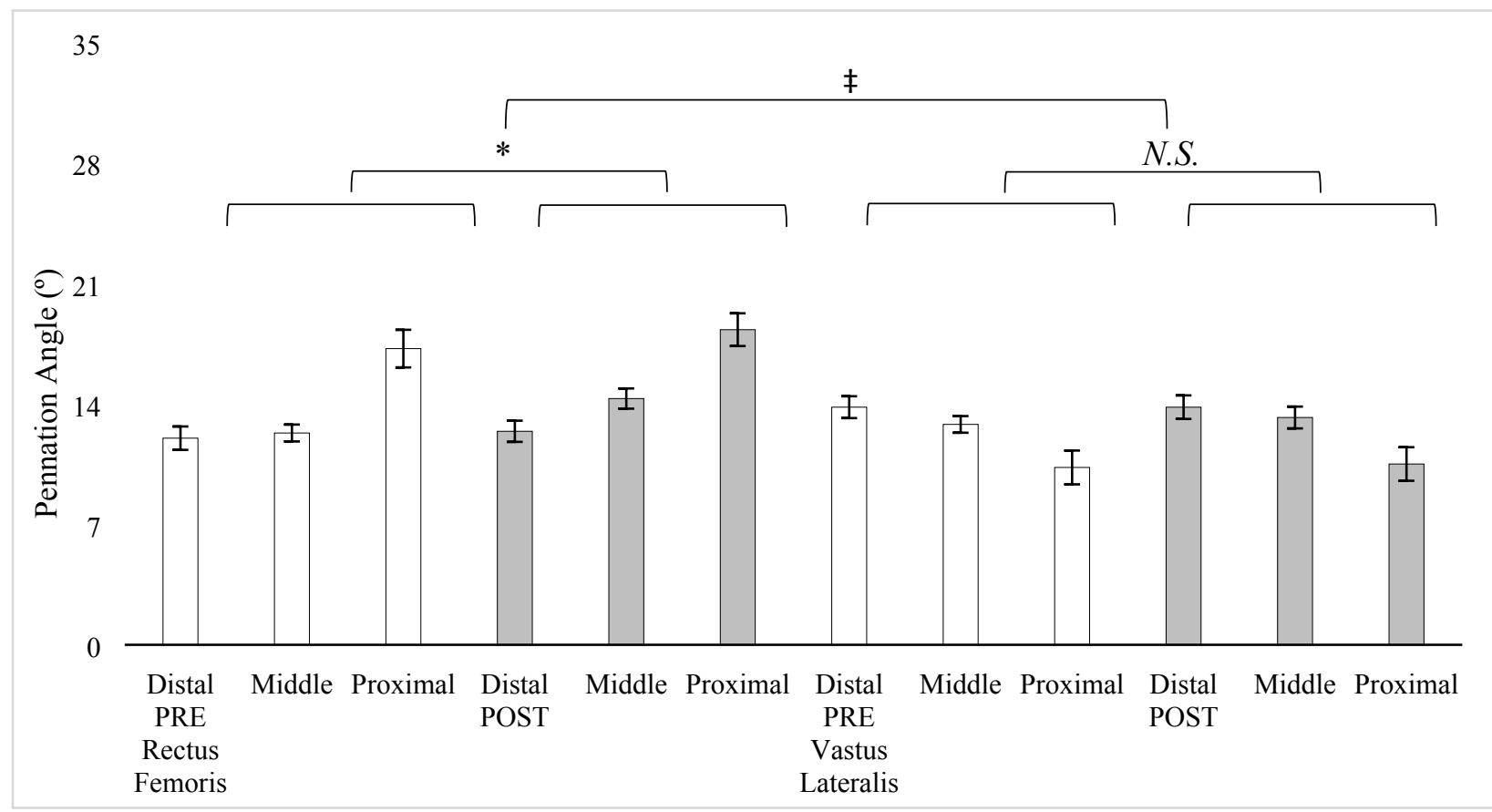

E.

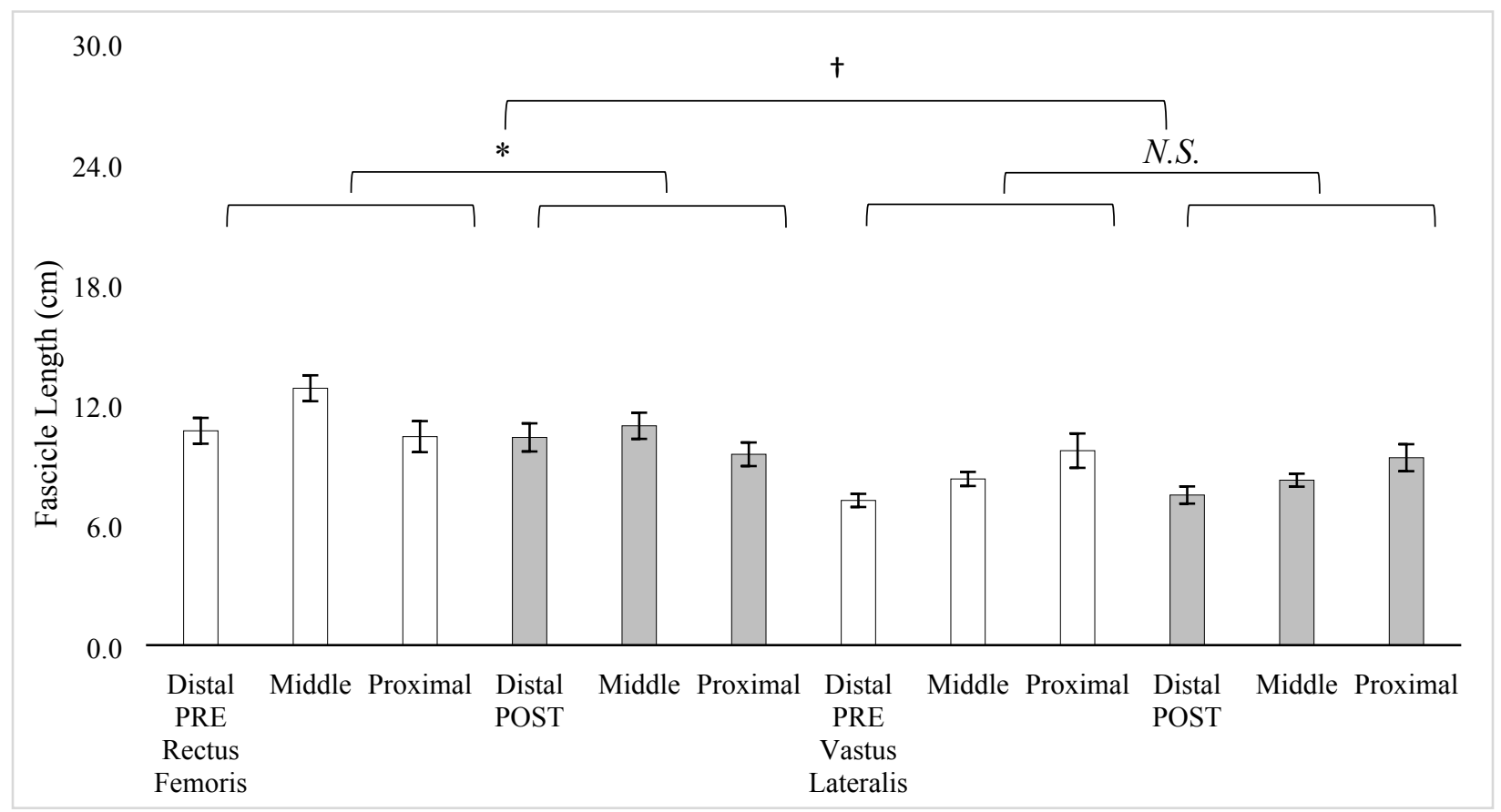

Note: $*=$ Significant $(\mathrm{p}<0.05)$ difference between PRE and POST; $\uparrow=$ Significant $(\mathrm{p}<0.05)$ differences between RF and VL; \# = Difference $(p<0.10)$ between PRE and POST; $+=$ Difference $(\mathrm{p}<0.10)$ between RF and VL; N.S. = Not significant 